\title{
Anodization of Glassy Carbon Electrodes in Oligomers of Ethylene Glycol and Their Monomethyl Ethers as a Tool for the Elimination of Protein Adsorption
}

\author{
Hatsuo Maeda, Munenori Itami, Kazunori Katayama, Yuji Yamauchi \\ and Hidenobu OHMORI
}

Faculty of Pharmaceutical Sciences, Osaka University, Yamada-oka, Suita, Osaka 565, Japan

\begin{abstract}
The adsorption property of bovine serum albumin (BSA) on glassy carbon (GC) electrodes anodized in $\mathrm{HO}\left(\mathrm{CH}_{2} \mathrm{CH}_{2} \mathrm{O}\right)_{n} \mathrm{R}$ $(n=1-4, \mathrm{R}=\mathrm{H}$ or $\mathrm{Me}$ ) has been evaluated by comparing cyclic voltammograms of caffeic acid before and after a treatment of the electrodes with a BSA solution. Electrodes modified with triethylene glycol and its monomethyl ether have proved to resist surface fouling due to BSA adsorption as well as to show satisfactory electrochemical performance. The reliability of the assessment was established by examining the amounts of BSA adsorbed on electrodes estimated by radioactivity measurements after a treatment with ${ }^{125}$ I-BSA. The analytical utility of a triethylene glycol-modified GC electrode has been demonstrated by analyzing a sample containing uric acid, acetaminophen, and BSA by reversed-phase high-performance liquid chromatography with an electrochemical detector, where a commercially available packedcolumn, allowing the passage of proteins, namely, requiring no pretreatment of samples for the removal of proteins, was utilized.
\end{abstract}

Keywords Protein adsorption, glassy carbon electrode, oligo(ethylene glycol), modified electrode, serum albumin, cyclic voltammetry, high-performance liquid chromatography

It is well known that the electrochemical analysis of biological samples is hampered by electrode fouling arising from non-specific protein adsorption, although many biologically important organic compounds are electrochemically detectable without rather troublesome derivatization. In order to circumvent this problem, many efforts have been devoted to the development of modification methods which increase the surface hydrophilicity, and thus decrease protein adsorption. Polymeric coating has proven to be a feasible technique for this purpose. ${ }^{1-8}$ However, polymer-coated electrodes seem to find only few applications ${ }^{2,7}$, except in stripping analysis of trace metals ${ }^{3-6,8}$, probably due to the slow mass transport of organic analytes in the membrane. Thus, electrodes with the desired surface conditions, prepared without relying on polymer chemistry, hold great promise for the electrochemical analysis of biological samples. Especially, the development of electrodes which resist protein adsorption will serve to draw out the potentiality of reversed-phase high-performance liquid chromatography (HPLC) using an electrochemical detector as a useful tool for the routine analysis of clinical samples. This is because columns packed with stationary phases which allow proteins to pass through have recently become commercially available for reversed-phase HPLC, and thus the removal of proteins from samples is no longer a necessary pretreatment for the HPLC analysis of biological fluids. Yet, only two studies on such modified electrodes have been reported.9,10

Recently, Whitesides et al. demonstrated that gold surfaces covered with mixed self-assembled monolayers containing $\mathrm{HS}\left(\mathrm{CH}_{2}\right)_{11}\left(\mathrm{OCH}_{2} \mathrm{CH}_{2}\right)_{n} \mathrm{OH}$ resist protein adsorption, depending on the mole fraction and the $n$ of the thiols in the interface; ${ }^{11-13}$ the thiol even with $n=2$ induces a significant prevention of adsorption. ${ }^{13}$ The results indicate that covering with poly(ethylene glycol) is not required to construct surface-resisting protein adsorption. Namely, the problem associated with protein adsorption can be solved just by affixing oligo(ethylene glycol) covalently, probably via ether-linkage, on an electrode surface. Such surface conditions will be easily realized on a glassy carbon (GC) electrode, based on our findings that GC electrodes are anodically modified via an ether-linkage, not only with simple 1-alkanols ${ }^{14,15}$, but also with $1, \omega$-alkanediols. ${ }^{16}$ We have thus examined the adsorption property of bovine serum albumin (BSA) on the surfaces of GC electrodes anodized in $\mathrm{HO}\left(\mathrm{CH}_{2} \mathrm{CH}_{2} \mathrm{O}\right)_{n} \mathrm{R}(n=1-4, \mathrm{R}=\mathrm{H}$ or $\mathrm{Me})$, electrodes 1-7 depicted in Fig. 1, by cyclic voltammetry. In this study, a GC electrode modified with triethylene glycol is shown to resist the protein adsorption as well as to exhibit satisfactory electrochemical performance; also its analytical utility has been demonstrated in reversedphase HPLC with the column mentioned above. In this context, Osa et al. recently reported that the anodization of GC electrodes in diols containing water can eliminate 


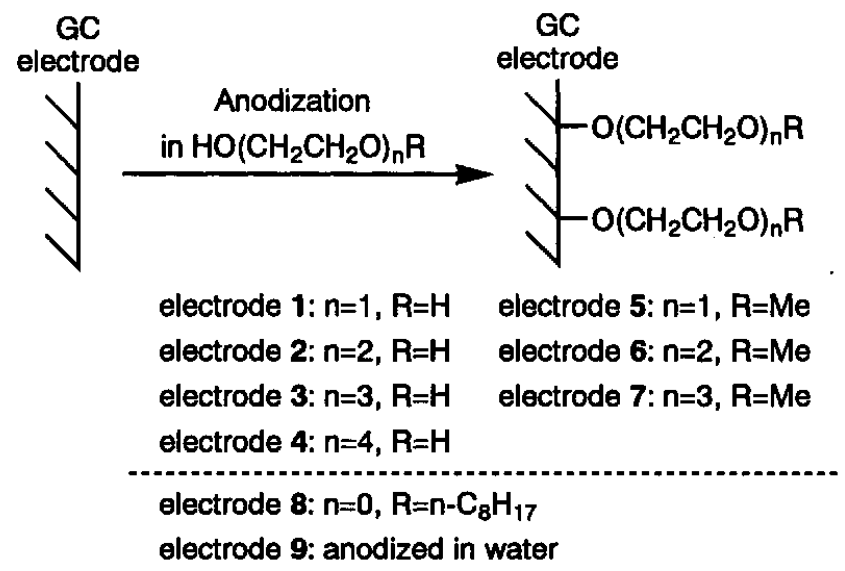

Fig. 1 Electrochemically modified GC electrodes used in this study.

the adsorption of human serum albumin. ${ }^{17}$ However, their modification method, which was slightly different from our original one ${ }^{14}$, seems to induce not only the fixation of the alkanol molecules, but also the increment of oxygen functionalities on the surfaces, allowing a possibility that their observation cannot be attributed merely to a modification with diols.

\section{Experimental}

\section{Materials}

BSA was purchased from Sigma Chemical Co. (St. Louis, MO, USA). BSA labeled with ${ }^{125}$ I was obtained as a buffer solution $(1.34 \mathrm{mCi} / \mathrm{mg}, 1.66 \mathrm{mCi} / \mathrm{ml})$ from ICN Pharmaceuticals, Inc. (Irvine, CA, USA). Deionized and distilled water was used throughout. All other chemicals were of reagent grade and used without further purification. A phosphate buffer $(0.1 \mathrm{M}, \mathrm{pH} 7.0$; $\mathrm{Na}_{2} \mathrm{HPO}_{4}+\mathrm{NaH}_{2} \mathrm{PO}_{4}$ ) was used for all experiments unless otherwise mentioned. A BSA solution at $\mathrm{pH} 3.6$ or 5.0 was prepared from an acetate buffer $(0.1 \mathrm{M}$; $\mathrm{CH}_{3} \mathrm{CO}_{2} \mathrm{H}+\mathrm{CH}_{3} \mathrm{CO}_{2} \mathrm{Na}$ ).

\section{Apparatus}

A GC electrode was polished with a polishing system (Model ML-150P, Maruto, Tokyo, Japan). An electrochemical modification of a GC electrode was performed by a potentiostat/galvanostat (Model HA 301, Hokuto Denko, Tokyo, Japan) connected to a coulomb/ amperehour meter (Model HF201, Hokuto Denko). Cyclic voltammograms were obtained with a potentiostat (Model 315A, Huso, Tokyo, Japan) equipped with an XY recorder (Model F-5C, Riken Denshi, Tokyo, Japan). A three-electrode configuration was employed: a GC disk $\left(7.07 \mathrm{~mm}^{2}\right)$ electrode with or without an electrochemical modification as the working electrode, a saturated calomel electrode (SCE) as the reference electrode, and a platinum wire as the counter electrode. GC rods (GC $30,3 \mathrm{~mm}$ i.d.) were obtained from Tokai carbon (Tokyo,
Japan). The fabrication of a GC electrode has been described previously. ${ }^{18}$ Radioactivity measurements were carried out with a liquid scintillation analyzer (TriCarb 1600CA, Packard Instrument Co., Inc., CT, USA), in which a GC plate (GC $30,10 \times 40 \times 3 \mathrm{~mm})$ purchased from Tokai Carbon was used. The HPLC system consisted of a pump (LC-10AD, Shimadzu, Kyoto, Japan), an on-line degasser (Model KT-17, Showa

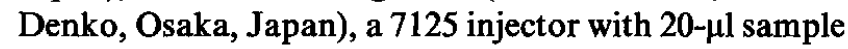
loop (Rheodyne, Cotati, CA, USA), a separation column (Wakopak Wakosil-GP-N6, $250 \times 4.6 \mathrm{~mm}$ i.d., Wako Pure Chemical Industries Ltd., Osaka, Japan), an electrochemical detector (L-ECD-6A, Shimadzu), and a recorder (C-R2A, Shimadzu). The working electrode for the electrochemical cell was a GC plate (GC 20, $15 \times 30 \mathrm{~mm}$ ) from Shimadzu.

\section{Electrode modification and measurement}

All of the modified GC electrodes were prepared by our revised method: ${ }^{19}$ a GC electrode was subjected to controlled-potential electrolysis in one of the oligomers containing $\mathrm{LiClO}_{4}(0.1 \mathrm{M})$ at $2.0 \mathrm{~V} v s$. $\mathrm{Ag}$ wire, where $5 \mathrm{mC}, 2 \mathrm{C}$, and $0.1 \mathrm{C}$ of electricity had been allowed to be consumed, to obtain electrodes for voltammetry, radioactivity measurements, and HPLC, respectively. Cyclic voltammetry was carried out for a solution of caffeic acid $(1.5 \mathrm{mM})$ in a phosphate buffer $(0.1 \mathrm{M}$, $\mathrm{pH}$ 7.0) at a bare or modified GC electrode before and after the electrode had been treated with $0.01 \%(w / v)$ BSA in a phosphate buffer (0.1 M, pH 7.0) for $5 \mathrm{~min}$ and rinsed with water, unless otherwise mentioned in the text. All of the voltammetric measurements were performed at room temperature and a scan rate of $0.1 \mathrm{~V} \mathrm{~s}^{-1}$. The radioactivity measurements with the $\gamma$-counter were performed on bare and modified GC plates after being exposed to a solution prepared from $20 \mathrm{ml}$ of a $0.1 \%$ (w/ v) BSA phosphate buffer solution (0.1 M, pH 7.4) and $20 \mu \mathrm{l}$ of the purchased ${ }^{125}$ I-BSA solution, and washed with a phosphate buffer $(0.1 \mathrm{M}, \mathrm{pH}$ 7.4) several times. HPLC was carried out using a phosphate buffer $(0.1 \mathrm{M}$, $\mathrm{pH}$ 7.0) as the mobile phase at room temperature.

\section{Results and Discussion}

After examining various markers to monitor BSA adsorption by cyclic voltammetry, caffeic acid (CA) (Fig. 2) has turned out to work the best, satisfying the following requirements: a satisfactory voltammetric response at a modified electrode; no fouling of the electrode merely by repetitive measurements; and clear indication for the well-known BSA adsorption to a bare GC surface. Figure 3 compares cyclic voltammograms of a CA solution in a phosphate buffer $(0.1 \mathrm{M}, \mathrm{pH} 7.0)$ at a bare GC electrode and electrode 3. CA exhibited a reversible redox wave at a bare electrode (Fig. 3a) and a partially retarded response at the modified electrode (Fig. 3c). As far as the amperometric detection of organic compounds in biological fluids is concerned, the 
<smiles>O=C(O)C=Cc1ccc(O)c(O)c1</smiles>

Fig. 2 Chemical structure of caffeic acid (CA).

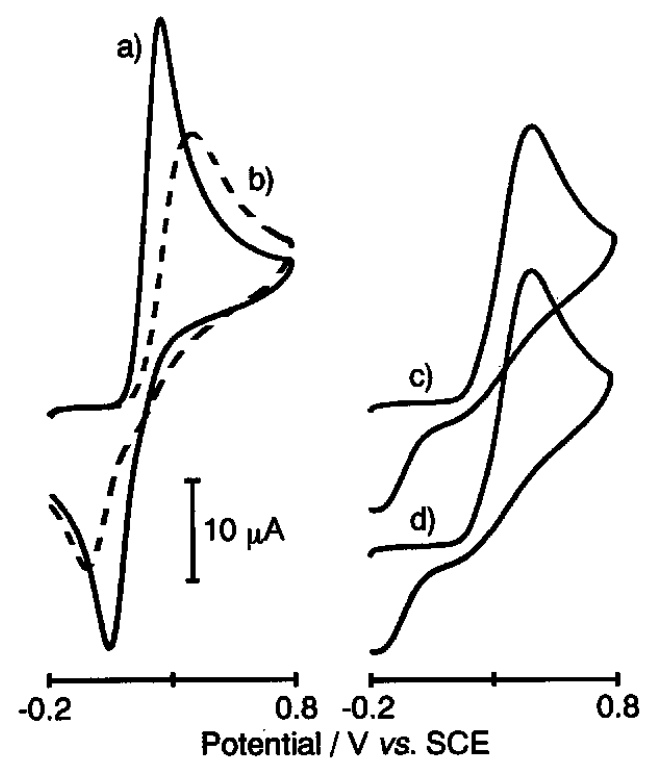

Fig. 3 Cyclic voltammograms of caffeic acid $(1.5 \mathrm{mM})$ in a phosphate buffer $(0.1 \mathrm{M}, \mathrm{pH} 7.0)$ at a bare GC electrode ( $a$ and $b$ ) and electrode 3 ( $c$ and d) before ( $a$ and $c$ ) and after (b and d) the treatment of the electrodes with $0.01 \%(w / v)$ BSA in a phosphate buffer $(0.1 \mathrm{M}, \mathrm{pH} 7.0)$ for $5 \mathrm{~min}$ : potential sweep rate, $0.1 \mathrm{~V} \mathrm{~s}^{-1}$.

electrochemical performance of electrode 3 seems to be quite satisfactory, despite the observation of poor reversibility in the voltammogram of CA. The irreversible response of CA induced by the modification could be attributed to a change in the $\mathrm{pH}$ in the close vicinity of the surface of electrode 3 , as previously proposed concerning phenomena observed on the voltammograms of various catechols at a polymer-coated electrode. ${ }^{7}$ After a treatment with $0.01 \%(\mathrm{w} / \mathrm{v}) \mathrm{BSA}$ in a phosphate buffer $(0.1 \mathrm{M}, \mathrm{pH}$ 7.0) for $5 \mathrm{~min}$, the electrode reaction of $\mathrm{CA}$ was apparently suppressed at the bare electrode (Fig. 3b), while no voltammetric change was observed at electrode 3 (Fig. 3d). The phenomenon recognized at the bare electrode will be ascribed to decrease in the effective surface area of the electrode, that is, a reduction in the amount of CA molecules diffusing to the electrode surface, due to the adsorption of albumin molecules. The results indicated that electrode 3 resists the adsorption of protein, as expected.

The effects of the conditions for a treatment with a BSA buffer solution, such as the time, protein concentration, and $\mathrm{pH}$, on the adsorption behavior of the protein on a bare electrode and electrode 3 were then

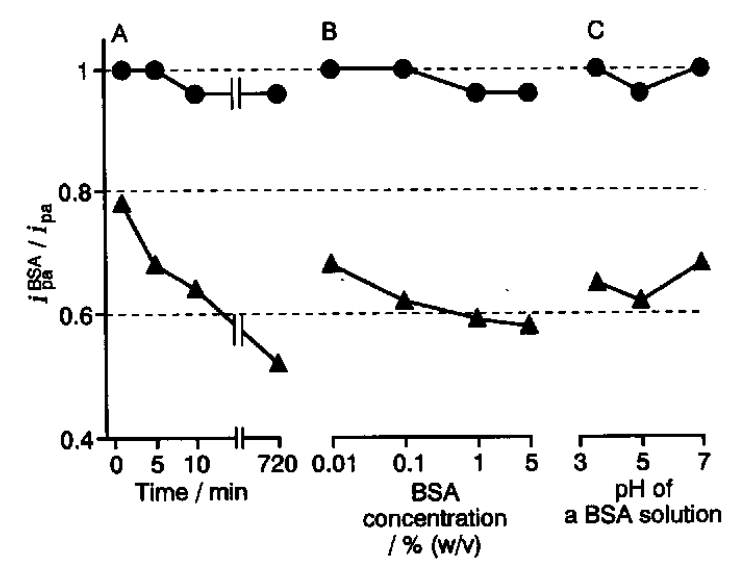

Fig. 4 Adsorption property of BSA on a bare GC electrode (triangles) and electrode 3 (circles) indicated by changes $\left(i_{\mathrm{pa}}^{\mathrm{BSA}} / i_{\mathrm{pa}}\right)$ in the voltammetric responses of caffeic acid $(1.5 \mathrm{mM})$ in a phosphate buffer $(0.1 \mathrm{M}, \mathrm{pH} 7.0)$ before and after the BSA treatment under various conditions: general conditions, with $0.01 \%(w / v)$ BSA in a phosphate buffer (0.1 M, pH 7.0) for $5 \mathrm{~min}$.

examined. In order to evaluate the adsorption behavior of BSA, the changes in the peak current $\left(i_{\mathrm{pa}}^{\mathrm{BSA}} / i_{\mathrm{pa}}\right)$ for the anodic wave of CA induced by the BSA treatment were used: $i_{\mathrm{pa}}$ and $i_{\mathrm{pa}}^{\mathrm{BSA}}$ denote the anodic peak currents for CA observed in a phosphate buffer at $\mathrm{pH} 7.0$ at each electrode before and after the treatment, respectively. The results are summarized in Fig. 4. The treatment of electrode 3 with $0.01 \%(\mathrm{w} / \mathrm{v}) \mathrm{BSA}$ in a phosphate buffer $(0.1 \mathrm{M}, \mathrm{pH} 7.0)$ for $12 \mathrm{~h}$ instead of $5 \mathrm{~min}$ resulted in only a negligible decrease in $i_{\mathrm{pa}}^{\mathrm{BSA}} / i_{\mathrm{pa}}$, while further suppression in the voltammetric response of CA was recognized at a bare electrode exposed to the BSA solution for a prolonged time (Fig. 4A). As for the concentration of BSA in a treatment for $5 \mathrm{~min}$, results similar to the case of the immersing time were observed on both electrodes: electrode 3 showed good resistance, regardless of the protein concentration; the surface of a bare electrode was more fouled as treated with a higher concentration of BSA (Fig. 4B). Changes in the pH of the media used for the treatment with $0.01 \%(\mathrm{w} / \mathrm{v})$ BSA for $5 \mathrm{~min}$ induced results with similar trends in $i_{\mathrm{pa}}^{\mathrm{BS}} / i_{\mathrm{pa}}$ at a bare electrode and electrode 3 , although the observed effect was smaller at the latter electrode. The BSA adsorption on each electrode seemed to be slightly accelerated at $\mathrm{pH} 5.0$ around the isoelectric point of BSA (Fig. 4C), which was reminiscent of $\mathrm{pH}$ effects on the adsorption behavior of BSA onto polystyrene latex ${ }^{20}$ and a bare gold electrode. ${ }^{21}$

The results described so far demonstrated that the treatment of electrodes with $0.01 \%(w / v) ~ B S A$ in a phosphate buffer $(0.1 \mathrm{M}, \mathrm{pH} 7.0)$ for $5 \mathrm{~min}$ can be regarded as typical and those conditions are reliable enough to assess the BSA adsorption on their surfaces by cyclic voltammetry of CA in a phosphate buffer (pH 7.0). Thus, voltammetric measurements under the above conditions were carried out for electrodes $1-7$, by which 
Table 1 Cyclic voltammetric data ${ }^{a}$ of caffeic acid at bare and modified GC electrodes before and after a treatment with a BSA solution, and contact angle $(\theta)$ of water at the electrodes

\begin{tabular}{rcccccc}
\hline Run & Electrode & $E_{\mathrm{pa}} / \mathrm{V}$ & $i_{\mathrm{pa}} / \mu \mathrm{A}$ & $E_{\mathrm{pa}}^{\mathrm{BSA}}-E_{\mathrm{pa}} / \mathrm{V}$ & $i_{\mathrm{pa}}^{\mathrm{BSA}} / i_{\mathrm{pa}}$ & $\theta / \mathrm{deg}$ \\
\hline 1 & bare & 0.23 & 38 & 0.13 & 0.68 & 66 \\
2 & $\mathbf{1}$ & 0.30 & 31 & 0.11 & 0.84 & 54 \\
3 & $\mathbf{3}$ & 0.46 & 26 & 0.03 & 0.96 & 50 \\
4 & $\mathbf{3}$ & 0.48 & 25 & 0.01 & 1.00 & 47 \\
5 & $\mathbf{6}$ & 0.24 & 39 & $-\mathrm{b}$ & $1.00^{\text {d }}$ & 40 \\
6 & $\mathbf{5}$ & 0.31 & 34 & 0.11 & 0.79 & 72 \\
7 & 6 & 0.43 & 28 & 0.06 & 0.91 & 62 \\
8 & 7 & 0.49 & 23 & -0.00 & 50 \\
9 & $\mathbf{8}$ & 0.22 & 56 & 0.01 & $0.57^{\mathrm{d}}$ & 95 \\
10 & 9 & & 0.91 & 0.91 & 43 \\
\hline
\end{tabular}

a. $E_{\mathrm{pa}}$ coupled with $i_{\mathrm{pa}}$, and $E_{\mathrm{pa}}^{\mathrm{BSA}}$ with $i_{\mathrm{pa}}^{\mathrm{BSA}}$ denote the potential ( $v s . \mathrm{SCE}$ ) and the current of the anodic peak observed on the voltammograms at the electrodes before and after the treatment, respectively: see text for details on cyclic voltammetry. b. No peak was observed. c, d. The value of $i_{\mathrm{pa}}$ or $i_{\mathrm{pa}}^{\mathrm{BSA}}$ was obtained as anodic current at $0.8 \mathrm{~V}$.

changes not only in $i_{\mathrm{pa}}^{\mathrm{BSA}} / i_{\mathrm{pa}}$ but also in the peak potential $\left(E_{\mathrm{pa}}^{\mathrm{BSA}}-E_{\mathrm{pa}}\right)$ for the anodic wave of $\mathrm{CA}$ induced by the BSA treatment were obtained: $E_{\mathrm{pa}}$ and $E_{\mathrm{pa}}^{\mathrm{BSA}}$ stand for the values for the anodic peak potential observed before and after the treatment, respectively. The results are summarized in Table 1, which also includes the contact angle $(\theta)$ of water on the electrodes.

As is clear from the results in runs $2-4, E_{\mathrm{pa}}^{\mathrm{BSA}}-E_{\mathrm{pa}}$ decreased and $i_{\mathrm{pa}}^{\mathrm{BSA}} / i_{\mathrm{pa}}$ approached unity as a GC electrode was modified with $\mathrm{HO}\left(\mathrm{CH}_{2} \mathrm{CH}_{2} \mathrm{O}\right)_{n} \mathrm{H}$ having a larger $n$. The modification, even with ethylene glycol, reduced the amount of BSA adsorption compared to the case of a bare GC electrode (runs 1 and 2). The resisting abilities of the modified electrodes showed a good relationship with their wettabilities: the modified electrode with a smaller $\theta$, that is, with a more hydrophilic surface, resisted BSA adsorption more strongly. However, electrode 4 with the most hydrophilic surface among the modified electrodes examined here was not appropriate for an electroanalytical purpose; CA exhibited no anodic peak at electrode 4 at all (run 5). Thus, a GC electrode anodized in triethylene glycol will have a surface that not only resists BSA adsorption, but also possibly attains a satisfactory response of electroactive organic compounds in biological samples.

The results in runs $6-8$ show the effects of substitution of a terminal hydroxyl group on electrodes $1-3$ with a methyl ether upon the adsorption property of BSA on the surfaces. The voltammetric data at electrodes 5-7 showed a similar trend to that observed at electrodes 1 3 ; the changes in the anodic responses of CA upon exposure to a BSA solution decreased as the number of ethylene oxide units in the modifier became larger; that is, the electrode surface became more hydrophilic, indicating that the elimination of BSA adsorption requires the presence not of a terminal hydroxy group, but of an ethylene oxide unit in the modifier, which is consistent with the findings by Whitesides et al. ${ }^{11}$ Among electrodes 5-7, electrode 7 has proven to effectively resist adsorption with a satisfactory electro-

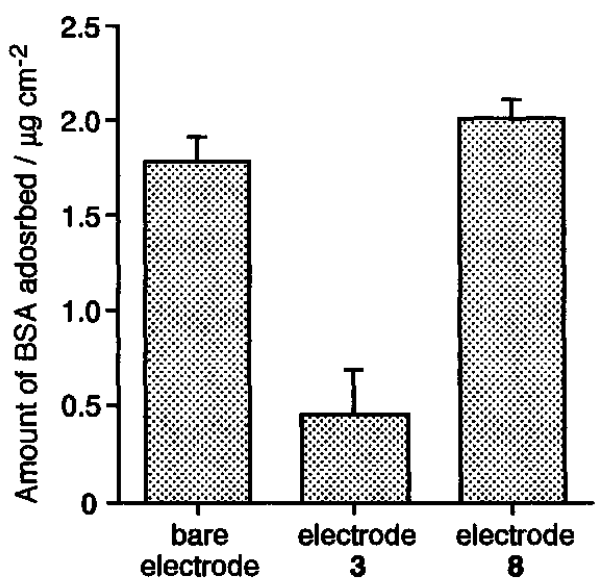

Fig. 5 Amount of BSA adsorbed on a bare GC electrode, and electrodes 3 and 8 estimated by the radioactivity measurements after the treatment of the electrodes with ${ }^{125}$ I-BSA: see text and experimental for details.

chemical sensitivity as electrode 3 .

In order to confirm the role played by the hydrophilicity of the electrode surfaces in eliminating protein adsorption, similar experiments were performed on GC electrodes anodized in 1-octanol and water (electrodes 8 and 9 in Fig. 1, respectively); electrode 8 has a more hydrophobic surface than does a polished GC electrode ${ }^{19}$, which was expected to enhance BSA adsorption on the surface; electrode 9 resists BSA adsorption to some extent, based on the facts that the anodization of GC electrodes in water results in the formation of a more hydrophilic surface than just being polished, which has recently been shown to be a useful way to prevent BSA adsorption on a GC electrode. 9 As expected, CA exhibited no anodic peak on the voltammogram between 0 and $0.8 \mathrm{~V}$ at electrode 8 after being treated with BSA, and the response even at $0.8 \mathrm{~V}$ was $57 \%$ of the anodic peak current observed before the treatment (run 9). A comparison of the contact angles of water (runs 1, 4, 8, and 
10) demonstrated that electrode 9 has a more hydrophilic surface, than not only the bare electrode, but also electrodes 3 and 7. However, the anodic treatment in water did not prevent BSA from adsorbing on the electrode surface as much as anodization in triethylene glycol and its monomethyl ether, although BSA adsorption was reduced on electrode 9 compared to that on a bare electrode. Thus, it has been demonstrated that the resistance to BSA adsorption depends not only on the hydrophilicity of the surfaces of the modified electrodes, but also on the size of the interfacial space enough to keep the protein out of the range tolerating its hydrophobic attraction to the electrode surface. This requirement is satisfied by the anodic modification of GC electrodes in triethylene glycol and its monomethyl ether.

The reliability of cyclic voltammetry of $\mathrm{CA}$ as a tool to evaluate the adsorption property of BSA on bare and modified GC electrodes was verified by the following non-electrochemical method, since $\mathrm{CA}$ has never been utilized as an electrochemical probe for the purpose, as far as we are aware. BSA adsorption on a bare GC electrode and electrodes 3 and 8 was compared by radioactivity observed for the electrodes after being exposed to a phosphate buffer solution of ${ }^{125}$ I-BSA. As can be seen in Fig. 5, the anodization of a GC surface in triethylene glycol remarkably reduced the BSA adsorption, the amount being about $25 \%$ of that before the modification; the amount of BSA adsorbed was greatest on electrode $\mathbf{8}$, which had been anodically modified with 1-octanol. The observed trend is well in line with the tendency recognized concerning changes in the voltammetric responses of $\mathrm{CA}$ by a treatment with BSA, suggesting that the present electrochemical method using $\mathrm{CA}$ as a probe is a reliable and useful tool for evaluating the adsorption of the protein on electrode surfaces. Accordingly, we could draw the conclusion that the anodization of GC surfaces in triethylene glycol and its monomethyl ether is a prominent way to prepare electrodes available for the electrochemical analysis of biological samples containing proteins.

To examine the reusabilities of electrodes 3 and 7 , a voltammetric measurement followed by a treatment with the BSA solution for $5 \mathrm{~min}$ was repeated. Such examinations are generally required in studies concerning the development of novel modified electrodes for analytical purposes. Figure 6 compares changes in the anodic peak current of CA observed at the modified electrodes and a bare electrode. Each response at electrode 3 was quite reproducible, and the original sensitivity was retained after 10 times of the BSA treatment. The electrochemical performance of electrode 7 was slightly retarded as the treatment with BSA was repeated, and the loss in sensitivity was $7 \%$ after 10 repetitions. This indicates that a modification with triethylene glycol rather than its monomethyl ether minimizes the BSA adsorption and/or accumulation of the reaction products on the electrode surfaces during successive electrochemical measurements more effectively. Immersing 10 times a bare GC electrode to the BSA buffer

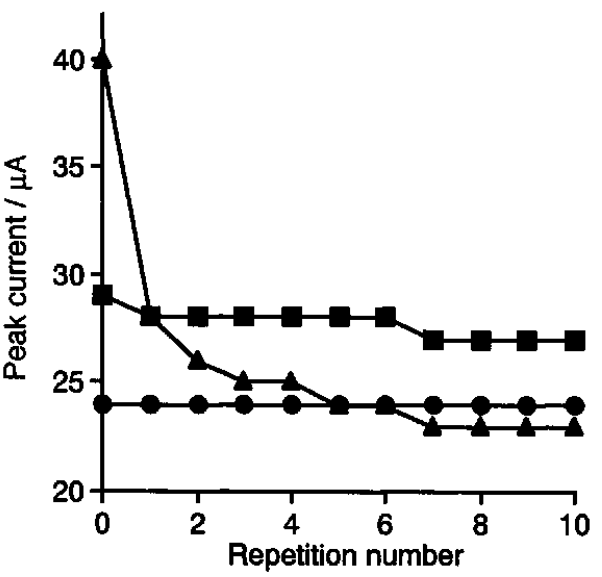

Fig. 6 Changes in the voltammetric responses of caffeic acid $(1.5 \mathrm{mM})$ in a phosphate buffer $(0.1 \mathrm{M}, \mathrm{pH} 7.0)$ at a bare GC electrode (triangles), electrode 3 (circles), and electrode 7 (squares) by a repetitive treatment of the electrodes with $0.01 \%(w / v)$ BSA in a phosphate buffer $(0.1 \mathrm{M}, \mathrm{pH} 7.0)$ for $5 \mathrm{~min}$.

solution resulted in a $43 \%$ loss in the anodic response of CA. This gave a peak current slightly smaller than that at electrode 3, although the original response at a bare electrode was 1.7-times as large as that observed after a modification with triethylene glycol.

The analytical utility of electrode 3 was demonstrated in a reversed-phase HPLC analysis of a sample containing BSA with an electrochemical detector, where a commercially available packed-column, allowing the passage of proteins, that is, requiring no pretreatment to remove proteins from samples, was utilized. As model analytes, uric acid and acetaminophen were chosen, since the flow injection analysis of the compounds was previously studied on electrodes coated with hydrophilic polymers. ${ }^{2,7}$ As an electrode for the electrochemical detection system, a GC plate had to be used, which has a much larger area than the electrodes for the voltammetric measurements. The amount of electricity suitable for anodizing the GC plate in triethylene glycol was estimated first, since the numbers of oligomer molecules fixed on the electrode surface, namely, the resistance against BSA adsorption as well as the electrochemical performance of the modified electrode, are controlled by the amount of electricity.

For the evaluation, hydrodynamic voltammograms at a bare GC plate electrode were compared with those at the electrodes anodized in triethylene glycol at various amounts $(0.1,0.5,1.0 \mathrm{C})$ of electricity, where HPLC was carried out for uric acid or acetaminophen $(1 \mu \mathrm{M})$ in a phosphate buffer (0.1 M, pH 7.0) under the following conditions: sample injection volume, $20 \mu \mathrm{l}$; mobile phase, phosphate buffer $(0.1 \mathrm{M}, \mathrm{pH} 7.0)$; flow rate, $1.0 \mathrm{ml} / \mathrm{min}$. Figure 7 shows the voltammograms obtained at a bare GC electrode and electrode 3 prepared at the expense of $0.1 \mathrm{C}$ of electricity. The results indicated that the modified electrode shows a satisfactory electrochemical 


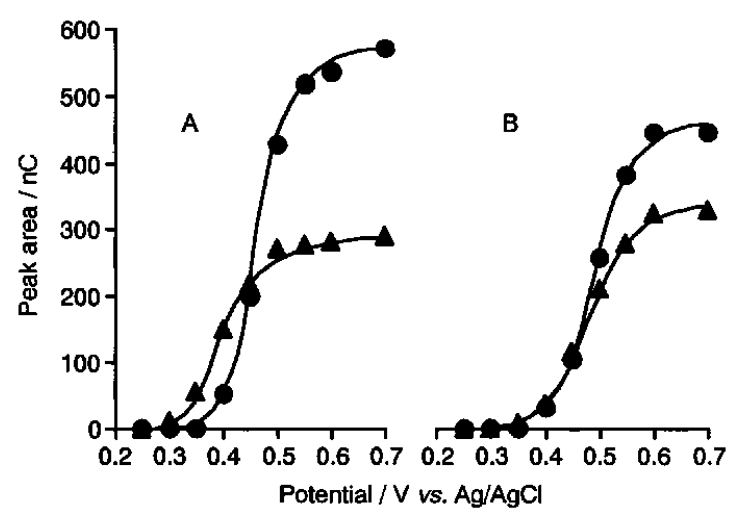

Fig. 7 Hydrodynamic voltammograms of $1 \mu \mathrm{M}$ uric acid (triangles) and $1 \mu \mathrm{M}$ acetaminophen (circles) at a bare electrode (A) and electrode 3 (B). HPLC conditions: mobile phase, phosphate buffer $(0.1 \mathrm{M}, \mathrm{pH} 7.0)$; flow rate, $1.0 \mathrm{ml} / \mathrm{min}$; column, Wakosil-GP-N6 (250×4.6 mm i.d.); injection volume, $20 \mu \mathrm{l}$.

performance in the flow system, although the modification induced a slight shift of $E_{1 / 2}$ to a more positive potential for both uric acid and acetaminophen, and a slight decrease in the plateau current for the latter. At electrode 3 , afforded by passing 0.5 or $1.0 \mathrm{C}$ of electricity in the electrochemical modification, the hydrodynamic voltammograms for uric acid and acetaminophen were remarkably retarded, their values of $E_{1 / 2}$ being more than $0.6 \mathrm{~V}$. Thus, a GC plate electrode modified with triethylene glycol by an anodic treatment at $0.1 \mathrm{C}$ of electricity turned out to be appropriate from the viewpoint of high sensitivity at the low applied potential required in electrochemical detection with HPLC.

A sample containing $1 \mu \mathrm{M}$ uric acid, $1 \mu \mathrm{M}$ acetaminophen, and $0.1 \%(\mathrm{w} / \mathrm{v}) \mathrm{BSA}$ in a phosphate buffer $(0.1 \mathrm{M}$, pH 7.0) was subjected to HPLC analysis with the thusobtained electrode 3 or a bare GC electrode as a detector; an applied potential was set at 0.45 or $0.6 \mathrm{~V}$. Figure 8 summarizes the results upon 10 repetitive injections of the sample; also, chromatograms obtained at $0.6 \mathrm{~V}$ are shown in Fig. 9.

In the detection at $0.45 \mathrm{~V}$ (open marks in Fig. 8), the sensitivity observed for both uric acid and acetaminophen at electrode 3 was less than half that at a bare GC electrode. However, surface fouling due to BSA adsorption was recognized at a bare electrode, and the responses for both analytes decreased as injection of the sample was repeated. In contrast, stable responses were afforded at the modified electrode, the relative standard deviations (RSDs) being $2.8 \%$ for uric acid and $9.5 \%$ for acetaminophen. When the applied potential was set at $0.6 \mathrm{~V}$ (closed marks in Fig. 8), the effects of BSA adsorption on the detection using a bare electrode seemed not to be as appreciable as those at $0.45 \mathrm{~V}$, judging from the responses for acetaminophen (RSD, $3.0 \%$ ). Yet, a considerably broad peak, probably due to anodic oxidation of BSA, appeared on the chro-

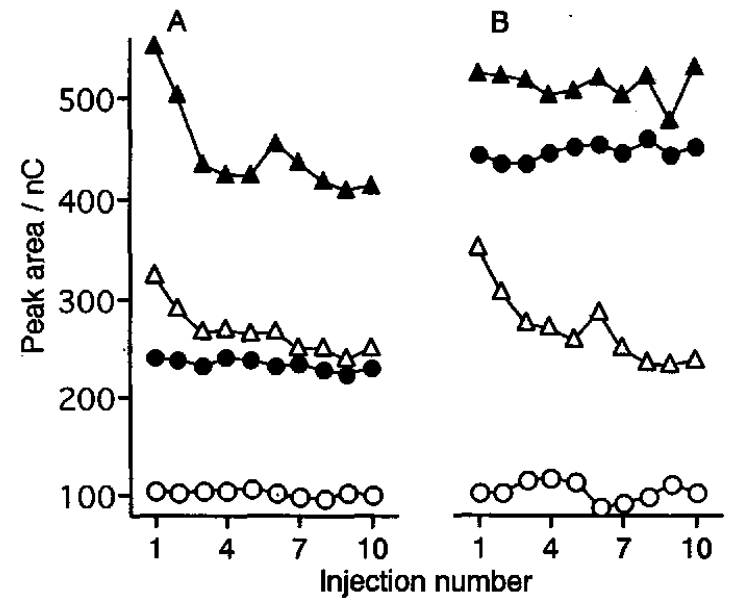

Fig. 8 HPLC results for 10 repetitive injections of a sample containing $1 \mu \mathrm{M}$ uric acid (A), $1 \mu \mathrm{M}$ acetaminophen (B), and $0.1 \%(\mathrm{w} / \mathrm{v}) \mathrm{BSA}$ in a phosphate buffer $(0.1 \mathrm{M}, \mathrm{pH} 7.0)$ with a bare GC electrode (triangles) and electrode 3 (circles) as a detector. HPLC conditions: applied potential, 0.45 and $0.60 \mathrm{~V}$ vs. $\mathrm{Ag} / \mathrm{AgCl}$ (open and closed marks, respectively); others given in Fig. 7.

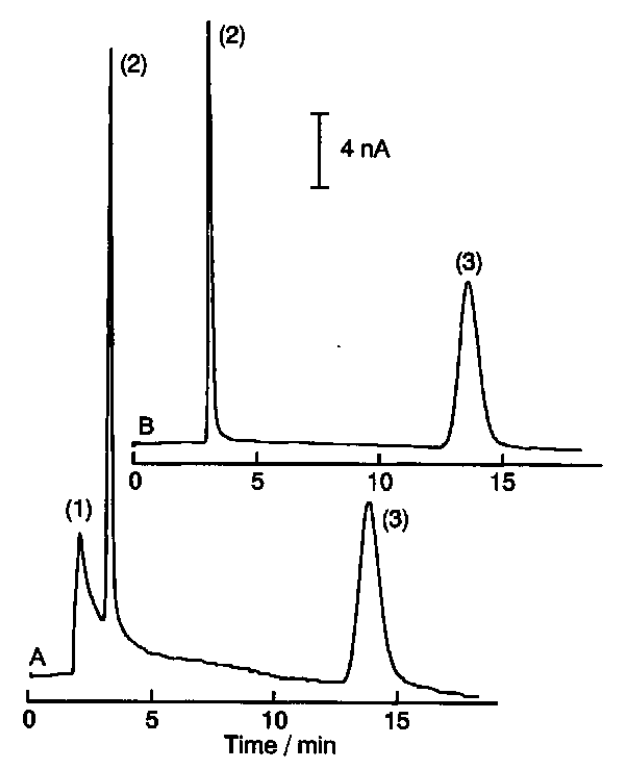

Fig. 9 Chromatograms of a sample containing $1 \mu \mathrm{M}$ uric acid, $1 \mu \mathrm{M}$ acetaminophen, and $0.1 \%(\mathrm{w} / \mathrm{v})$ BSA in a phosphate buffer $(0.1 \mathrm{M}, \mathrm{pH} 7.0)$ with a bare electrode (A) and electrode 3 (B) as a detector. Peaks: 1, BSA; 2, uric acid; 3, acetaminophen. HPLC conditions: applied potential, $0.6 \mathrm{~V}$ vs. $\mathrm{Ag} / \mathrm{AgCl}$; others given in Fig. 7 .

matograms obtained under the conditions (Fig. 9A), by which reliability in the observed responses, especially for uric acid, seemed to be lost to some extents. Utilizing electrode 3 in place of a bare GC electrode as a detector prevented the electrochemical reaction of BSA at $0.6 \mathrm{~V}$ from giving rise to an undesired broad peak (Fig. 9B), and allowed detection with high reproducibil- 
ity for both uric acid and acetaminophen, the RSDs being 2.6 and $1.8 \%$, respectively. Although the present modification brought about some decrease in the responses, the extent seems to be relatively small compared to the reported results on flow injection analysis at polymer-coated GC electrodes: the loss in sensitivity in the detection of acetaminophen, $13 \%$ at $0.6 \mathrm{~V} v s . \mathrm{Ag} /$ $\mathrm{AgCl}$ with electrode $3 ;>60 \%$ at $0.7 \mathrm{~V} v s$. $\mathrm{Ag} / \mathrm{AgCl}$ with an Eastman-AQ polymer coated electrode; ${ }^{7}>90 \%$ at $0.8 \mathrm{~V} v s . \mathrm{Ag} / \mathrm{AgCl}$ with a base-hydrolyzed cellulosic film coated electrode. ${ }^{2}$

The present results on HPLC have demonstrated the potentialities of a GC electrode anodized in triethylene glycol, not only to overcome the problems in the electrochemical analysis of samples containing proteins, but also to minimize the loss in sensitivity, which seems to be generally unavoidable at common modified electrodes. Further studies on a more practical application and improvement in the electrochemical performance of the modified electrode are now in progress.

We are grateful for the financial support for Shimadzu Science Foundation.

\section{References}

1. G. Sittampalam and G. S. Wilson, Anal. Chem., 55, 1608 (1983).

2. J. Wang and L. D. Hutchins, Anal. Chem., 57, 1536 (1985).

3. J. Wang and L. D. Hutchins-Kumar, Anal. Chem., 58, 402 (1986).

4. B. Hoyer, T. M. Florence and G. E. Batley, Anal. Chem., 59, 1608 (1987).
5. B. Hoyer and T. M. Florence, Anal. Chem., 59, 2839 (1987).

6. J. Wang and Z. Lu, J. Electroanal. Chem. Interfacial Electrochem., 266, 287 (1989).

7. J. Wang and T. Golden, Anal. Chem., 61, 1397 (1989).

8. J. Wang and Z. Taba, Electroanalysis, 2, 383 (1990).

9. A. J. Doward and A. D. Roddick, Electroanalysis, 6, 409 (1994).

10. A. J. Doward and A. D. Roddick, Electroanalysis, 7, 376 (1995).

11. K. L. Prime and G. M. Whitesides, Science [Washington, D.C.], 252, 1164 (1991).

12. C. Pale-Grosdemange, E. S. Simon, K. L. Prime and G. M. Whitesides, J. Am. Chem. Soc., 113, 12 (1991).

13. K. L. Prime and G. M. Whitesides, J. Am. Chem. Soc., 115 10714 (1993).

14. H. Maeda, Y. Yamauchi, M. Hosoe, T.-X. Li, E. Yamaguchi, M. Kasamatsu and H. Ohmori, Chem. Pharm. Bull., 42, 1870 (1994).

15. H. Maeda, M. Hosoe, T.-X. Li, M. Itami, Y. Yamauchi and H. Ohmori, Chem. Pharm. Bull., 44, 559 (1996).

16. H. Maeda, Y. Yamauchi, M. Yoshida and H. Ohmori, Anal. Sci., 11, 947 (1995).

17. B. Guo, J. Anzai and T. Osa, Chem. Pharm. Bull., 44, 860 (1996).

18. H. Ohmori, A. Matsumoto and M. Masui, J. Chem. Soc., Perkin Trans. 2, 1980, 347.

19. H. Maeda, M. Itami, Y. Yamauchi and H. Ohmori, Chem. Pharm. Bull., 44, 2294 (1996).

20. H. Shirahama, K. Takeda and T. Suzawa, J. Colloid Interface Sci., 109, 552 (1986).

21. J. Anzai, B. Guo and T. Osa, Chem. Pharm. Bull., 42, 2391 (1994).

(Received April 7, 1997)

(Accepted July 22, 1997) 